\title{
Cardioprotective Effect of Danshensu against Ischemic/Reperfusion Injury via c-Subunit of ATP Synthase Inhibition
}

\author{
Qing Gao, JingYi Zhao, Zixuan Fan, Jiadi Bao, \\ Dawei Sun, Huhu Li, Chun Sun, and Xijuan Jiang \\ School of Integrative Medicine, Tianjin University of Traditional Chinese Medicine, Tianjin 300193, China \\ Correspondence should be addressed to Xijuan Jiang; xijuanjiang@foxmail.com
}

Received 13 May 2017; Revised 2 August 2017; Accepted 18 October 2017; Published 8 November 2017

Academic Editor: Subash C. Gupta

Copyright (C) 2017 Qing Gao et al. This is an open access article distributed under the Creative Commons Attribution License, which permits unrestricted use, distribution, and reproduction in any medium, provided the original work is properly cited.

\begin{abstract}
Mitochondrial permeability transition pore (MPTP) opening is the main culprit of ischemic/reperfusion (IR) injury. It is reported that c-subunit of ATP synthase is the core component of MPTP. Danshensu (DSS), a monomer isolated from the traditional Chinese herb Danshen, has showed cardioprotective effect against IR injury through unknown mechanism. In this study, rat hearts were suspended in Langendorff instrument and perfused with Krebs-Henseleit (KH) buffer containing DSS for 60 minutes, followed by 30 minutes of global ischemia. Parameters including heart rate, left ventricular developed pressure, and the rate of left ventricle diastolic pressure change were recorded to assess their cardiac function. All these indexes were improved in DSS group. The rate of cardiomyocytes apoptosis and MPTP opening were both inhibited in DSS group. In addition, DSS administration leads to downregulation of c-subunit of ATP synthase in both mRNA and protein levels. Consistently, when c-subunit of ATP synthase was overexpressed in H9C2 cells through pcDNA3/5G1 plasmid transfection, MPTP opening was enhanced when the cardioprotective effect of DSS also tapers. In conclusion, DSS could alleviate cardiac IR injury via inhibiting c-subunit of ATP synthase expression.
\end{abstract}

\section{Introduction}

Although routine treatments for cardiac ischemia such as coronary bypass surgery, thrombolysis, and percutaneous coronary intervention have made satisfying outcome, the occurrence of ischemic/reperfusion (IR) injury should not be neglected [1]. Multiple reasons, such as oxidative stress, inflammation, and cell death, contribute to IR injury. Recent studies showed that mitochondrial permeability transition pore (MPTP) opening is the fundamental cause of IR injury [2]. MPTP is a nonspecific pore at the inner mitochondrial membrane whose opening is triggered by $\mathrm{Ca}^{2+}$ overload or oxidative stress. MPTP opening is a process that its inner membrane suddenly enables free diffusion of solutes up to $1.5 \mathrm{kDa}$ in size [3]. However, excessive MPTP opening will lead to inner membrane potential collapse, respiratory chain uncoupling, and halt of mitochondrial ATP synthesis. Eventually mitochondria become swelling and rupture and ensue with cell death [4]. Taking into consideration its role in reperfusion-mediated cardiac damage, MPTP opening is a prime target for therapies that aims at cardioprotection [5].

MPTP is considered to be a multiprotein complex includes the voltage-dependent anion channel at the outer membrane, adenine nucleotide translocator at the inner membrane, and cyclophilin D at the matrix [6,7], all of which are considered to be major modulators of MPTP. However, they are dispensable to the structure of MPTP $[8,9]$. Recent studies showed that $\mathrm{c}$-subunit of the $\mathrm{F}_{0}$ ATP synthase constitute a critical component of MPTP $[10,11]$. Multiple copies of c-subunit form a closed ring, which forms the ion-driven membrane rotors of ATP synthases. The csubunit comprises two trans-membrane helices, and its cring features an ion-binding site in paired adjacent subunits. Normally, the c-ring remains closed until the episode of $\mathrm{Ca}^{2+}$ overload or oxidative stress such as during IR process when it opens followed by opening of MPTP [12]. In contrast, $\mathrm{He}$ et al. reported persistence of MPTP opening in the absence of subunit $c$ of human ATP synthase [13]. The 
c-ring model of MPTP meets challenges. Although we cannot make sure whether c-subunit is the core of MPTP, altered cring of ATP synthase increases sensitivity of mitochondria to undergo MPTP opening as important determinants of the reduced tolerance to ischemic-reperfusion injury [14]. Therefore, inhibition of extravagant opening of c-ring could inhibit extravagant opening of MPTP.

Danshen is an herb medicine used in clinic to treat cardiovascular diseases, such as myocardial hypertrophy and ischemia reperfusion injury. Danshensu (DSS) is a major water-soluble active component of Danshen. The mechanism of DSS in treating IR is known to be through its antioxidants effect and ability to reduce $\mathrm{Ca}^{2+}$ overload [15-17]. Therefore, we proposed DSS might play a role in preventing the opening of MPTP. In the present study, we investigated the role of DSS in treating IR through manipulating the expression of c-ring of ATP synthase.

\section{Materials and Methods}

2.1. Drugs and Regents. DSS was provided by National Institutes for Food and Control, of purity of over 98\%. Cell Counting Kit-8 (CCK-8) kit was purchased from Dojindo (Rockville, MD, USA). Terminal-deoxynucleotidyl transferase mediated nick end labeling (TUNEL) kit was brought from Roche (St Louis, MO, USA). Mitochondria Isolation Kit was purchased from Beyotime Biotechnology Inc. (Beijing, China). MPTP kit (For mitochondrial and cell use) was purchased from GENMED (Shanghai, China). Tetrachlorotetraethyl benzimidazole carbocyanine iodide (JC-1) was purchased from Invitrogen (Thermo Fisher, Shanghai, China). Primary antibody against c-subunit and caspase-3 were purchased from Abcam (Cambridge, UK). Quantitative polymerase chain reaction (QPCR) kit was from Tiangen (Beijing, China). Plasmid was purchased from GeneChem (Shanghai, China).

2.2. Animals. Male Sprague-Dawley (SD) rats $(250 \pm 30 \mathrm{~g})$ were supplied by Experimental Animal Center of the Tianjin University of Traditional Chinese Medicine. Rats $(n=45)$ were randomly assigned to the following groups: control group, model group, and DSS group with 15 rats in each group. All procedures conform to the regulations that was stipulated by Animal Care Committee of Tianjin University of Traditional Chinese Medicine and to the Principles of Laboratory Animal Care that were formulated by the National Society for Medical Research and to the Guide for the Care and Use of Laboratory Animals that was published by the National Institute of Health (NIH Publication number 85-23, revised 1996).

\subsection{Langendorff Isolated Heart Perfusion Preparation. The} rats were anesthetized by chloral hydrate $(300 \mathrm{mg} / \mathrm{kg}$, ip). As soon as negative tail-flick response was confirmed, its chest was opened. The heart was rapidly harvested and then incubated in ice-cold Krebs-Henseleit (KH) buffer with aorta cannulated. Following Langendorff method, the heart was mounted on Langendorff apparatus and retrogradely

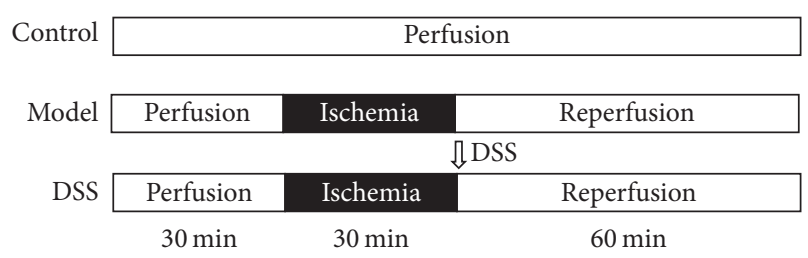

FIGURE 1: Scheme of IR experiment. The hearts were harvested from rats and then hung in the Langendorff apparatus. Control group was perfused with $\mathrm{KH}$ buffer for $120 \mathrm{~min}$. Model and DSS group first perfusion for $30 \mathrm{~min}$, then through $30 \mathrm{~min}$ ischemia, and $60 \mathrm{~min}$ reperfusion: $10 \mu \mathrm{M}$ DSS were added to $\mathrm{KH}$ buffer in the DSS group during reperfusion step.

perfused in vitro via aorta at the pressure of $70 \mathrm{mmHg}$. The perfusion medium was continuously bubbled with a mixture of $95 \% \mathrm{O}_{2}$ and $5 \% \mathrm{CO}_{2}$ at $37^{\circ} \mathrm{C}$, reaching the $\mathrm{pH}$ of 7.4 . Each heart was housed in a controlled heart chamber maintained at $37^{\circ} \mathrm{C}$. Coronary perfusion was applied by Langendorff system using $\mathrm{KH}$ buffer, containing $105.7 \mathrm{mM} \mathrm{NaCl}, 5.36 \mathrm{mM}$ $\mathrm{KCl}, 1.28 \mathrm{mM} \mathrm{CaCl}_{2}, 1.084 \mathrm{mM} \mathrm{MgCl}_{2}, 6 \mathrm{mM} \mathrm{H}_{2} \mathrm{O}, 1.18 \mathrm{mM}$ $\mathrm{KH}_{2} \mathrm{PO}_{4}, 18 \mathrm{mM} \mathrm{NaHCO}$, and $11 \mathrm{mM}$ Glucose. Control group was reperfused with $\mathrm{KH}$ continuously. In model and DSS groups, perfusion was stopped for 30 mins followed by reperfusion for $60 \mathrm{~min}$. DSS at $10 \mu \mathrm{M}$ [18] was added to $\mathrm{KH}$ solution in the DSS group (Figure 1). Then the heart tissues were used for the next experiments.

2.4. Cell Culture and Treatments. H9C2 cell line isolated from rats was used in our study. ATP5G1-overexpression plasmid was transfected by lipo2000 into H9C2 cell. The transfect efficiency was assessed by QPCR after $24 \mathrm{~h}$. Oxygen glucose deprivation (OGD) model was used to simulate ischemicreperfusion injury. In detail, cells were replaced with nonglucose Dulbecco's modified eagle medium (DMEM) and incubated in $95 \% \mathrm{~N}_{2}, 5 \% \mathrm{CO}_{2}$ atmosphere for three hours; then the cells were replaced with high-glucose DMEM containing $10 \% \mathrm{FBS}$ in $95 \% \mathrm{O}_{2}, 5 \% \mathrm{CO}_{2}$ for four hours.

Cells were divided into five groups: control, model, DSS, pcDNA3/5G1, and pcDNA3/5G1 + DSS group. In DSS and DSS + 5G1 group, $10 \mu \mathrm{M}$ DSS in the reoxygenation step was added. Cell viability, apoptosis, and MPTP opening status were detected by CCK-8 kit, TUNEL staining, and cell MPTP kit, respectively.

2.5. Cardiac Function Measurement and Observation. Cardiac function was evaluated by parameters including left ventricular developed pressure (LVDP), the max and minimum rate of left ventricle diastolic pressure change per min $( \pm \mathrm{dp} / \mathrm{dt})$, and heart rates (HR). LVDP equals left ventricle end systolic pressure minus by left ventricle end diastolic pressure. $\pm \mathrm{dp} / \mathrm{dt} \max$ is the maximal and minimum rate of pressure development. LVDP, $\pm \mathrm{dp} / \mathrm{dt} \max$, and HR were calculated from the LV pressure curve. These parameters were recorded continuously using Powerlab data acquisition system (8SP Chart 5 software; A.D. Instruments, Castle Hill, Australia) [19]. Part of left ventricular tissues were harvested and stored as frozen section for further TUNEL examination. 
Mitochondria isolated from heart tissue were used for MPTP opening and mitochondrial membrane potential (MMP) analysis. The remaining heart tissues were homogenate at $10 \%$ $(\mathrm{w} / \mathrm{v})$ and stored at $-80^{\circ} \mathrm{C}$ for further quantifying $\mathrm{c}$-subunit mRNA level and ATP synthase protein level.

2.6. TUNEL Examination. Terminal-deoxynucleotidyl transferase mediated nick end labeling (TUNEL) assay was used to evaluate apoptosis in ischemic reperfused heart tissue and H9C2 cell. Rat hearts were harvested and frozen-sectioned which were then processed under instruction of In Situ Cell Death Detection Kit, Fluorescein (Roche). For quantitative analysis, TUNEL-positive cells in five different slides from different hearts were counted. The apoptotic index was defined as the percentage of TUNEL-positive myocytes per slide.

2.7. MPTP Opening and MMP Detection. For in vivo study, mitochondria were isolated from heart tissue according to the protocol of Mitochondria Isolation Kit [20]. MPTP opening and mitochondrial membrane potential were then examined under instruction of Mitochondria MPTP fluorescence detection kit (For mitochondria use only) and tetrechlorotetraethylbenzimidazol carbocyanine iodide (JC-1) [21]. For in vitro study, MPTP opening was detected using cell MPTP fluorescence detection kit (For cell use only).

2.8. Quantitative Polymerase Chain Reaction (QPCR). Total RNA was isolated from the cardiac tissue using Trizol (invotrogen) and reversely transcribed into cDNA by superscript reverse transcriptase (Tiangen, Beijing, China) at $42^{\circ} \mathrm{C}$ for $1 \mathrm{~h}$. Reverse transcription material (100 ng) was amplified with Taq DNA polymerase (Tiangen, Beijing, China). Primers were synthesized by Sangon Biotech (Shanghai, China).

The primer pair specific to ATP 5G1 is as follows:

Forward sequence $5^{\prime}$-GGAGTGGGAGTGCAGATTGAA-3'

Reverse sequence $5^{\prime}$-TGGTACAGGAGCGGATCAGA $-3^{\prime}$

The primer pair specific to GAPDH is as follows:

Forward sequence $5^{\prime}$-GCATCTTCTTGTGCAGTGCC- $3^{\prime}$

Reverse sequence $5^{\prime}$-GATGGTGATGGGTTTCCCGT $-3^{\prime}$

Polymerase chain reaction (PCR) products of LOX-1 and GAPDH were 78 base pairs and 262 base pairs, respectively. Forty cycles $\left(94^{\circ} \mathrm{C}\right.$ for $30 \mathrm{~s} ; 60^{\circ} \mathrm{C}$ for $1 \mathrm{~min}$; and $72^{\circ} \mathrm{C}$ for $1 \mathrm{~min}$ ) were used in PCR reaction. Gene expression profile was analyzed by $2^{-\Delta \Delta C T}$ ([delta] [delta] CT ) method.

2.9. Western Blotting. Proteins were obtained from heart tissues after the whole perfusion process. Quantification of the protein was conducted using a modified bicinchoninic acid (BCA) assay (Cwbio, China). Protein samples were prepared by homogenizing rat heart, then being boiled for 5 minutes with loading buffer (Cwbio, China). Samples were separated using sodium dodecyl sulfate polyacrylamide gel electrophoresis (SDS-PAGE) on 15\% gel and transferred onto polyvinylidene fluoride (PVDF) membrane by electroblotting. After being blocked using skimmed milk for $1 \mathrm{~h}$, the membranes were incubated at $4^{\circ} \mathrm{C}$ overnight with the primary antibody. The following day, the membranes were washed and incubated with the goat anti-rabbit horseradish peroxidase-conjugated secondary antibody at room temperature for $1 \mathrm{~h}$. Protein bands were visualized using an enhanced chemiluminescence kit (Cwbio, China) under a ChemiDoc imaging system (Bio-Rad Laboratories, Berkeley, CA, USA).

2.10. Statistical Analysis. Each quantitative experiment was repeated three times. Statistical results were presented as means \pm SEM. $P$ values of $<0.05$ were denoted as statistically significant. One-way analysis of variance (ANOVA) was used to assess the differences among the groups. Fisher's least significant difference (LSD) was used in multiple comparisons between groups. Nonparametric statistical method of Mann-Whitney test was used to assess histopathology of myocardial damage level data. All statistical analyses were calculated using SPSS 17.0 (International Business Machines Corp., Armonk, NY, USA).

\section{Results}

3.1. Effect of DSS on Cardiac Function and Cardiomyocytes Apoptosis. The hearts were harvested from rats and then hung in the Langendorff apparatus. Cardiac function was recorded by the Powerlab/16s record software in Langendorff system through the whole reperfusion process. Control group was perfused with $\mathrm{KH}$ buffer for $120 \mathrm{~min}$. For model and DSS group, first perfusion for $30 \mathrm{~min}$, then through $30 \mathrm{~min}$ ischemia, and $60 \mathrm{~min}$ reperfusion: $10 \mu \mathrm{M}$ DSS was added to $\mathrm{KH}$ buffer in the DSS group during reperfusion step. The normal HR was within 250-300 BPM. During ischemia, HR declined rapidly to zero. HR was not recovered following reperfusion for $60 \mathrm{~min}$ in model group. On the other side, in DSS group, HR recovered to normal level after reperfusion for $20 \mathrm{~min}$. In control group, LVDP was maintained constantly at $50-80 \mathrm{mmHg}$. In model group, it was kept at $20-30 \mathrm{mmHg}$ in the reperfusion process On the contrary, LVDP recovered to normal level when heart undergoing reperfusion for $20 \mathrm{~min}$ in DSS group. Similarly, $\pm \mathrm{dp} / \mathrm{dt}$ index failed to recover to baseline in model group during the whole reperfusion process while it recovered to normal level when heart was reperfused for $20 \mathrm{~min}$ in the DSS group (Figure 2). These results indicated that DSS could improve cardiac function significantly in ischemic-reperfusion process.

Cardiomyocytes apoptosis was detected by TUNEL method and caspase-3 expression. As shown in Figures 3(a) and 3(b), cell apoptosis rate in model group was 3 times over the DSS group. As shown in Figures 3(c) and 3(d), caspase-3 expression in heart of model group was 2 times higher than the DSS group, which means DSS can alleviate cell apoptosis. 


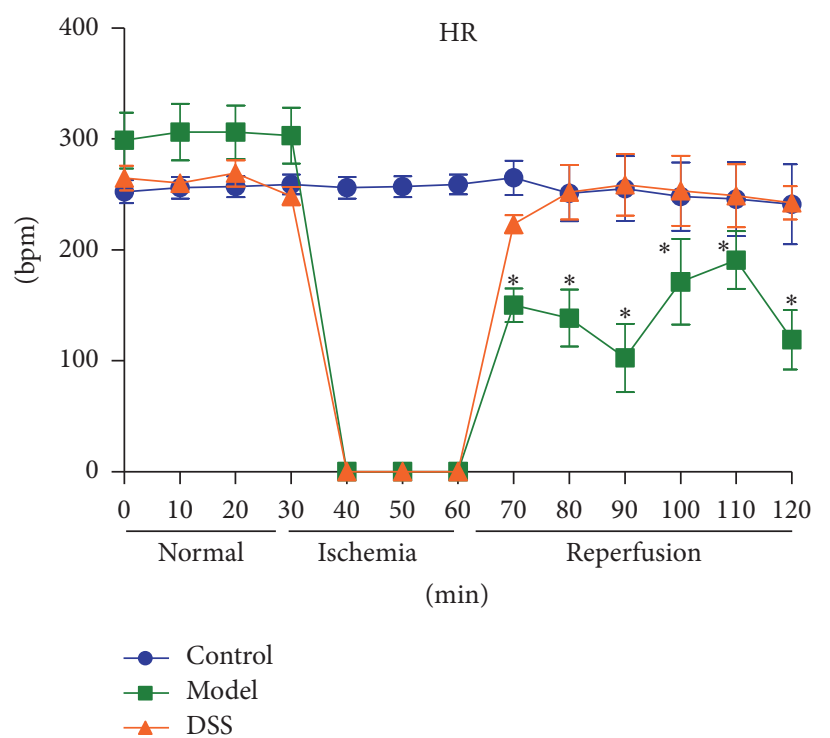

(a)

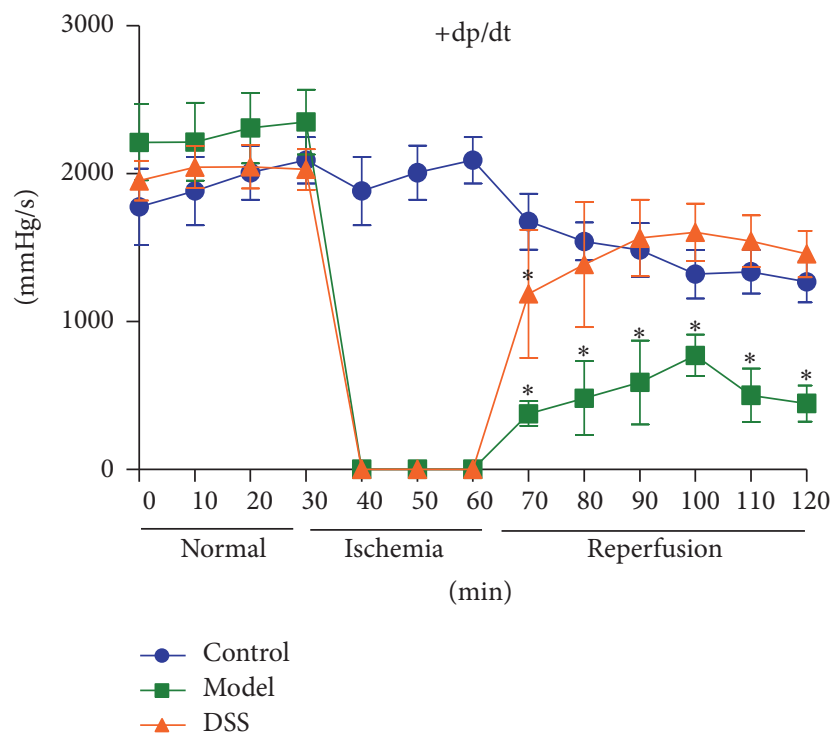

(c)

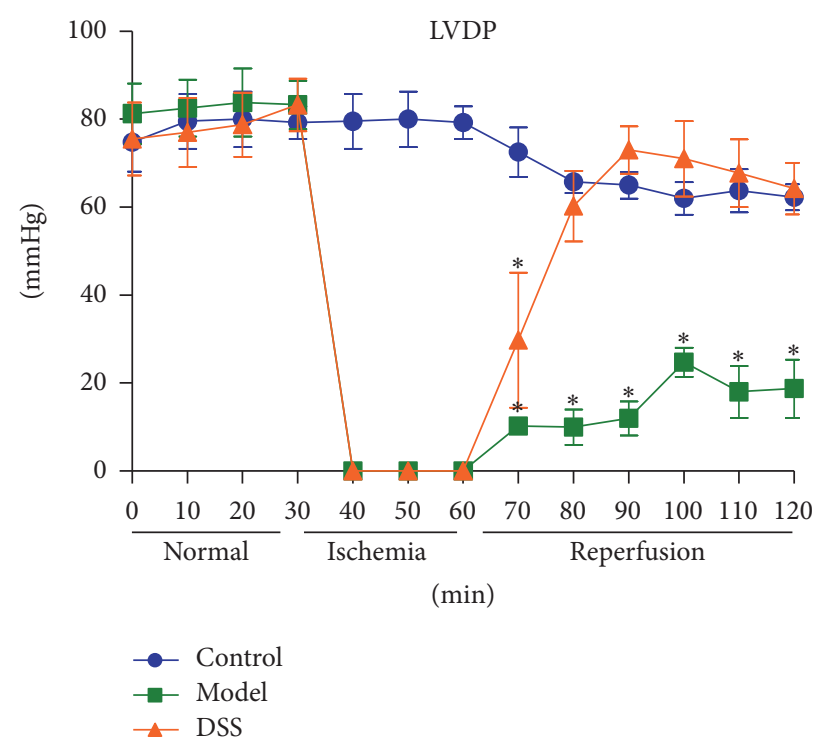

(b)

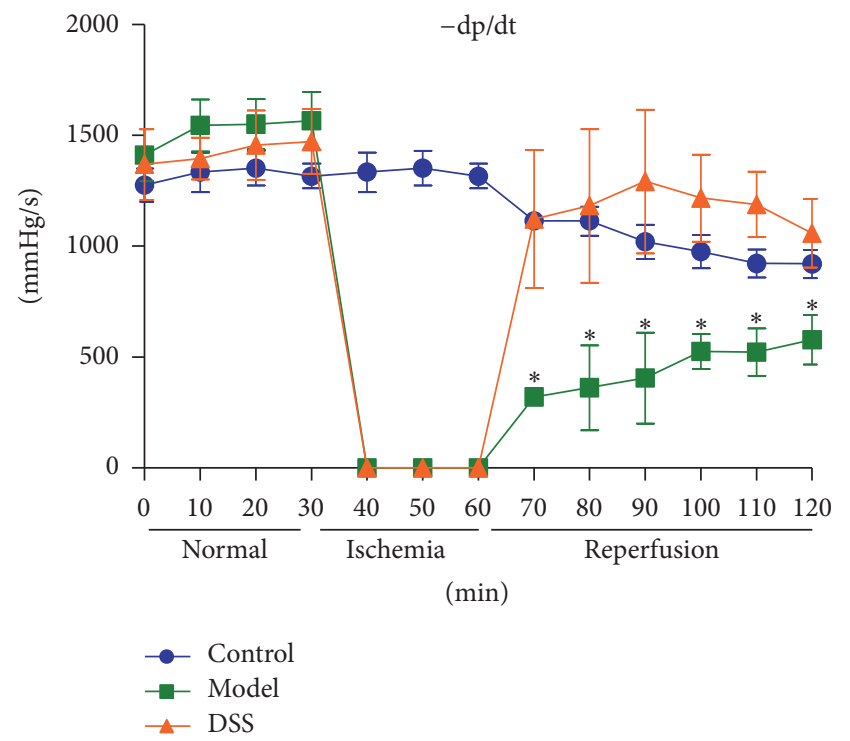

(d)

Figure 2: Cardiac function data recorded by Powerlab/16s software. After the hearts were hung in the Langendorff apparatus, Powerlab/16s software was used to collection the data of cardiac function. (a) Heart rate. (b) Left ventricular developed pressure (LVDP). (c) Development of left ventricular pressure maximum rising rate $(+\mathrm{dp} / \mathrm{dt})$. (d) Development of left ventricular pressure minimum decline rate $(-\mathrm{dp} / \mathrm{dt})$. Values were expressed as mean \pm SEM. ${ }^{*} P<0.05$, compared to control group.

3.2. Effect of DSS on the Opening of MPTP. When the reperfusion process finished, hearts samples were harvested. Mitochondria were isolated from heart tissue for MPTP opening detection. Mitochondria MPTP kit and JC-1 dye were used to evaluate the status of MPTP opening. Mitochondria MPTP kit used calcein-AM as fluorescence probe. When calceinAM entered into mitochondria, it was excised by lactonase to produce fluorescent calcein with strong polarity and then captured by mitochondria. At the same time, calcein outside mitochondria was quenched by cobalt ions. As soon as MPTP opens, calcein release from mitochondria can also be quenched by cobalt ions. Thus the relative fluorescence unit (RFU) changes in the mitochondria indicate MPTP opening.
MPTP opening in model group significantly enhanced compared to its intact level in DSS group. JC-1 was used to detect the mitochondrial membrane potential (MMP) changes, which reflect the degree of MPTP opening indirectly. Our results showed that MMP decreased significantly due to IR in model group compared to its unaltered level in DSS group (Figure 4). In a word, DSS can inhibit the MPTP opening.

3.3. Effect of DSS on the Expression of ATP c-Subunit. Total protein was obtained from heart tissue after the reperfusion process. C-subunit of ATP synthase expression in heart tissue was detected by western blotting. Expression of ATP 


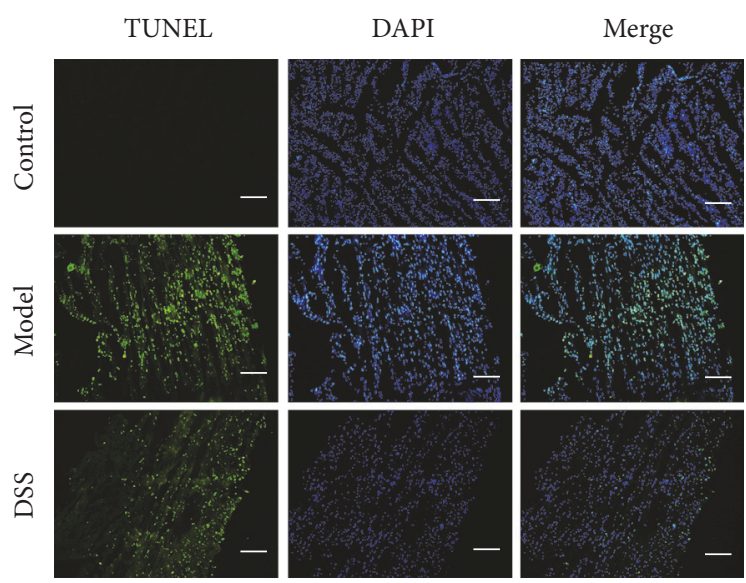

(a)

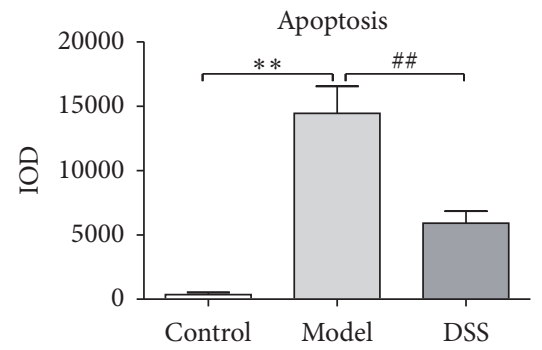

(b)

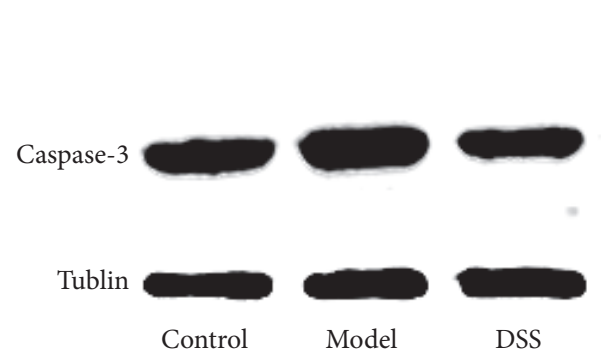

(c)

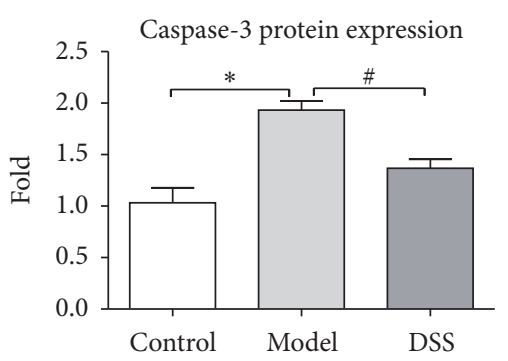

(d)

FIgURE 3: Cardiac apoptosis rate assay. (a) TUNEL detection. Nuclear was stained by DAPI and presents blue fluorescent in heart tissue. Apoptosis was detected by DNA strand breaks stained by TUNEL kit and present green fluorescent. Scale bar $=100 \mathrm{um}$. (b) Apoptosis rate was calculated by IPP software from five visual fields in each group. Apoptosis rate declined significantly in DSS group. (c) Western blot results of caspase-3 protein expression. (d) Statistic data of caspase-3 protein expression. Values were expressed as mean \pm SEM. ${ }^{*} P<0.05$, ${ }^{* *} P<0.01$, compared to control group. ${ }^{\#} P<0.05,{ }^{\#} P<0.01$, compared to model group.

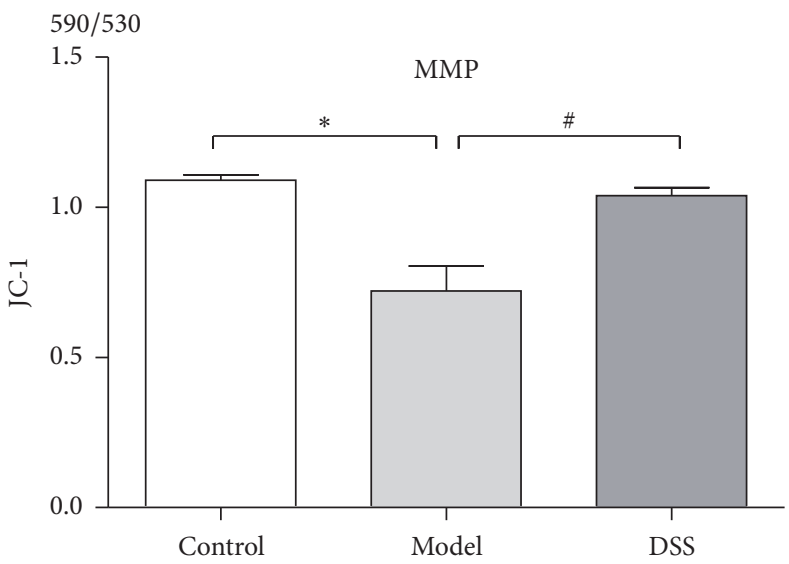

(a)

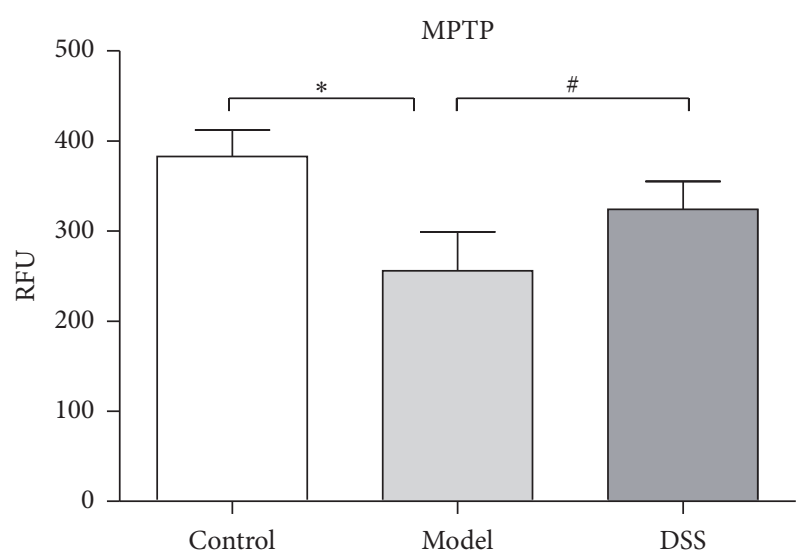

(b)

FIGURE 4: (a) Mitochondrial membrane potential (MMP) was detected by JC-1 dye. MMP declined significantly in model group and remained normal level in DSS group. (b) Mitochondrion permeability transition pore (MPTP) was detected by MPTP kit. RFU decline means MPTP opening degree upregulated. Thus, MPTP opening significantly upregulated in model group but not in DSS group. Values were expressed as mean \pm SEM. ${ }^{*} P<0.05$, compared to control group. ${ }^{\#} P<0.05$, compared to model group.

c-subunit was significantly upregulated after IR while it remained in normal level in DSS group (Figure 5). The results indicated that DSS inhibited MPTP opening possibly through inhibiting c-subunit of ATP synthase expression.
3.4. Effect of DSS in ATP c-Subunit Overexpressed H9C2 Cell Lines. H9C2 cells were transfected with pcDNA3/5G1 plasmid. To evaluate the transfection effect, both mRNA transcription level and protein expression level of ATP 5G1 


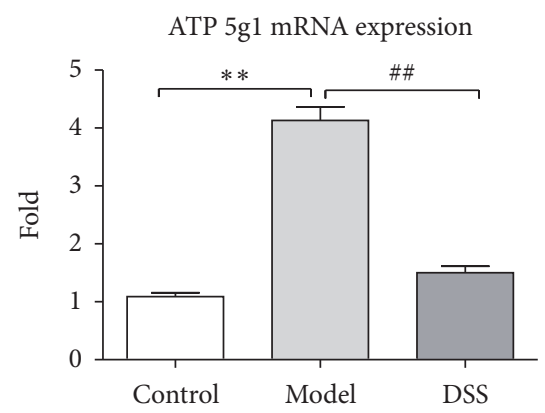

(a)



(b)

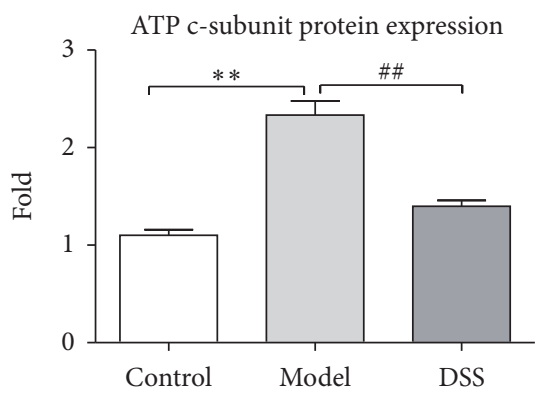

(c)

FIGURE 5: ATP 5G1 expression. (a) ATP 5G1 mRNA expression was detected by QPCR. (b) Western blot results of ATP 5G1 protein expression. (c) Statistic data of ATP 5 G1 protein expression. Values were expressed as mean \pm SEM. ${ }^{* *} P<0.01$, compared to control group. ${ }^{\# \#} P<0.01$, compared to model group.

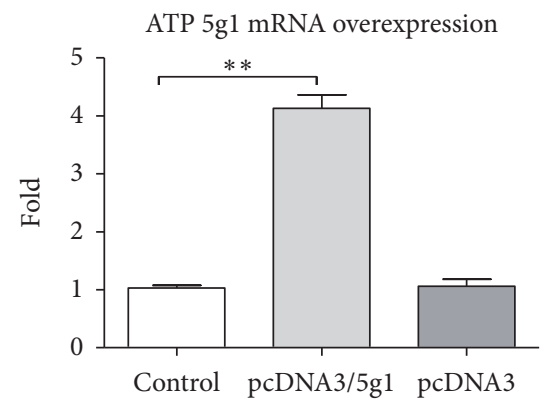

(a)

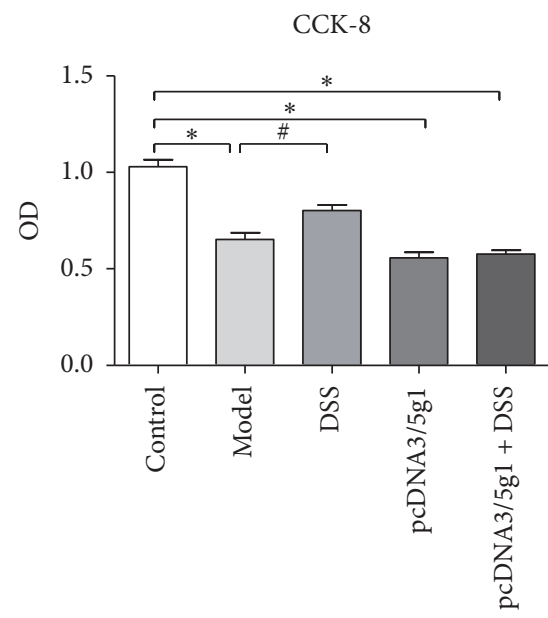

(d)

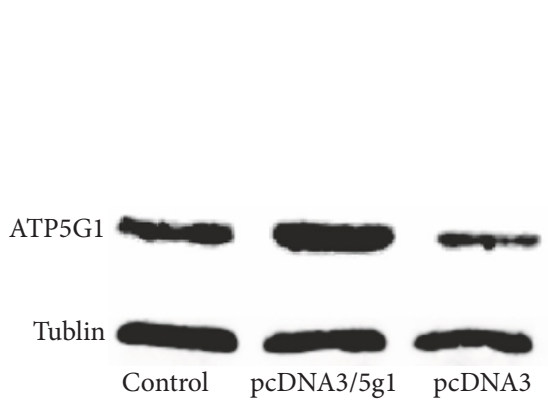

(b)

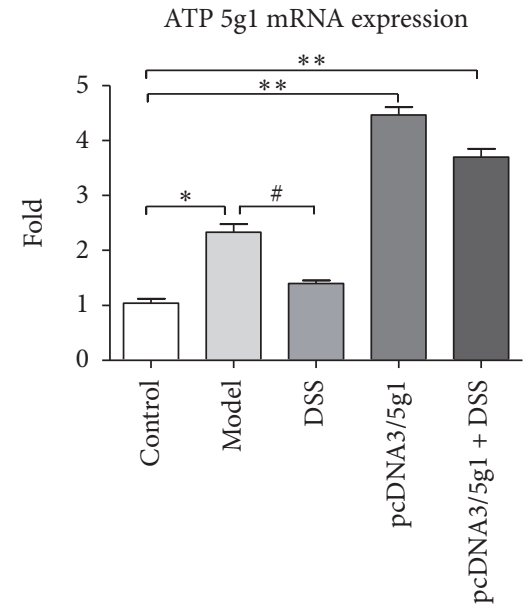

(e)

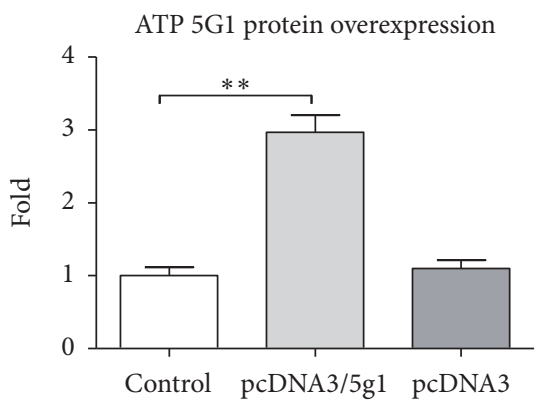

(c)

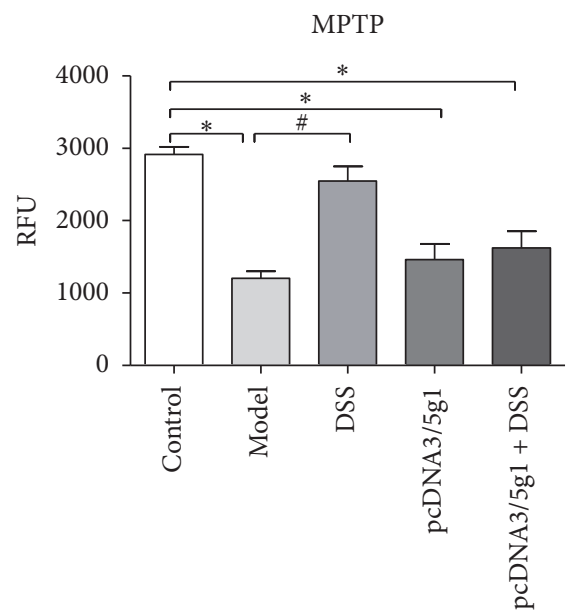

(f)

Figure 6: Cell experiments. (a-b) Transfection effect evaluation: (a) ATP 5G1 mRNA expression detected by QPCR after transfection experiment. (b) ATP 5G1 protein expression detected by western blotting after transfection experiment. (c) Statistic data of ATP 5G1 protein expression after transfection experiment. (d) Cell viability detected by CCK-8. (e) ATP 5G1 mRNA expression in each group. (f) MPTP opening detected by MPTP kit. Values were expressed as mean \pm SEM. ${ }^{*} P<0.05,{ }^{* *} P<0.01$, compared to control group. ${ }^{\#} P<0.05$, compared to model group.

were quantified. ATP $5 \mathrm{Gl}$ mRNA expression was four times higher than that of the nontransfection cell (Figure 6(a)). Consistently, ATP5G1 protein expression level was shown to be three times higher than that of nontransfection cell.

DSS was also shown to be able to improve cell vitality as indicated by CCK8 in all group but not in the pcDNA3/5G1 transfection group (Figure 6(d)). ATP 5G1 mRNA expression was upregulated in model group but not in DSS group, which insinuated that DSS can inhibit ATP 5G1 mRNA expression during IR injury (Figure 6(e)). DSS was also shown to be able to protect cells from apoptosis in control H9C2 cells but not in the plasmid transfection cells (Figure 7). MPTP opening 

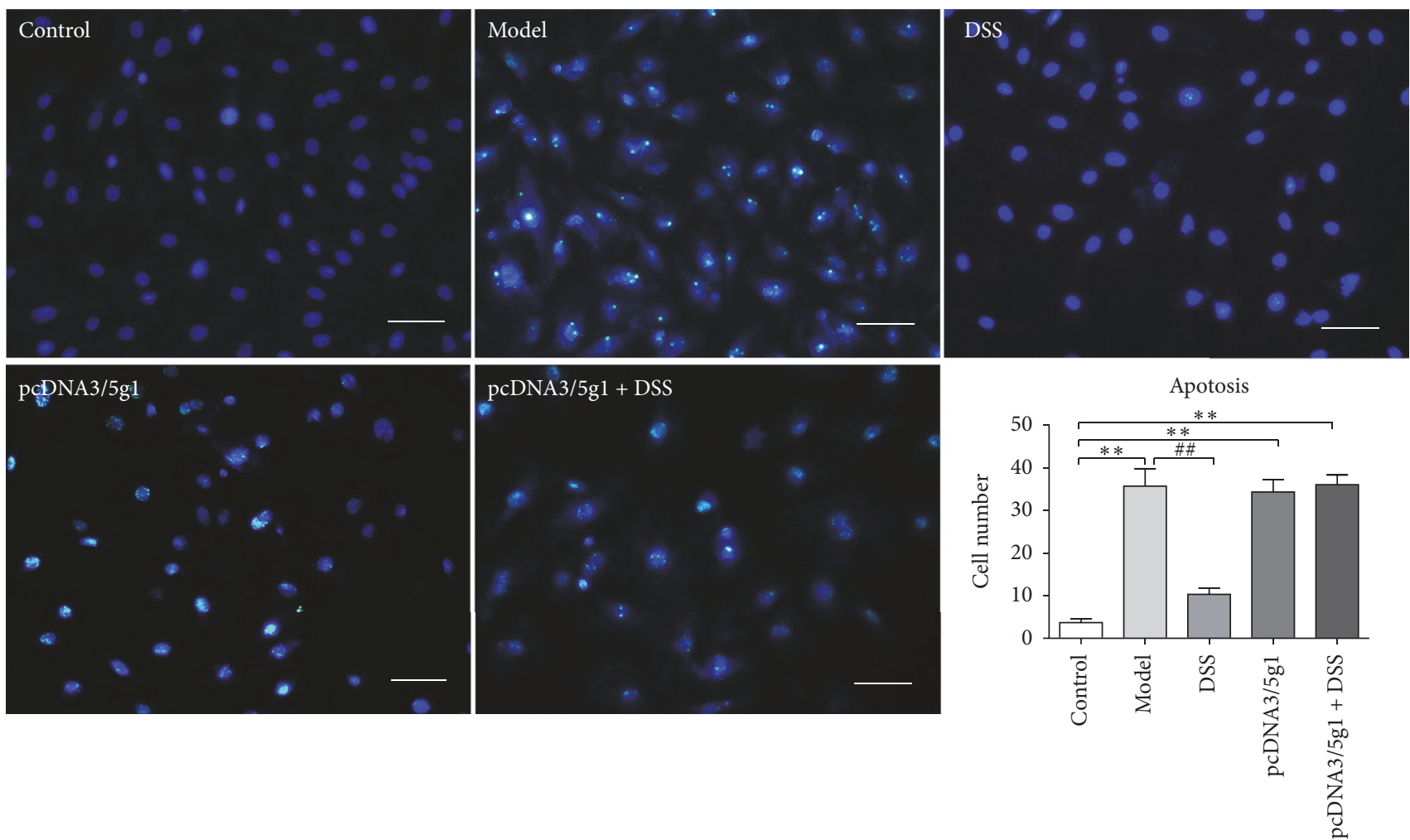

Figure 7: Cell apoptosis rate detected by TUNEL and DAPI. Nuclear was stained by DAPI and presents blue fluorescent. Apoptosis was detected by DNA strand breaks stained by TUNEL kit and presents green fluorescent. Scale bar $=50 \mathrm{um}$. Values were expressed as mean \pm SEM. ${ }^{* *} P<0.05$, compared to control group. ${ }^{\# \#} P<0.01$, compared to model group.

was also inhibited in control cells but not in the plasmid transfection group (Figure 6(f)). Therefore, DSS could protect cells from ischemic-reperfusion injury through inhibiting csubunit of ATP synthase expression, and the protection effect waned under circumstance of ATP 5G1 overexpression.

\section{Discussion}

MPTP opening plays a critical role during the episode of cardiac ischemic-reperfusion injury. The c-subunit of ATP synthase was used as target for new drug development [22]. Being a component in Chinese herb that was used widely in cardiovascular diseases, the underlying mechanism of DSS in treating IR injury still needs to be elucidated.

DSS, a water soluble isolated from Salvia miltiorrhiza (Danshen), has been widely used in clinic for the treatment of ischemic-reperfusion injury in China [23]. Several studies reported its effects in improving cardiac function both in vivo and in vitro, which are consistent with our results [15]. In this study, DSS improve cardiac function indicated by the fact that HR and LVDP were both upregulated significantly but were recovered soon after reperfusion in DSS group. What is more, it was shown that apoptosis rate declined significantly in DSS group followed IR injury both in vivo and in vitro. DSS could improve cell viability and decrease cell apoptosis rate in the OGD model.

Many studies explore the underlying mechanism of DSS in alleviating IR injury, such as its antioxidant and antiapoptotic effect and its role in reducing the calcium overload $[16,17,24]$, but no study has reported its role in MPTP opening under IR injury background. Previous studies revealed that MPTP opening is the main cause of cell death and cardiac damage [25]. MPTP has been considered as nonspecific pores, whose opening is responsible for the osmotic influx of water into the mitochondrial matrix and results in swelling of mitochondria and dissipation of the mitochondrial membrane potential with cell death ensuring [17]. Results showed that the degree of MPTP opening was upregulated with decreased MMP during IR. On the contrary, the degree of MPTP opening declined and MMP remains normal level with DSS treatment in episode of IR. Not surprisingly, the inhibition effect of DSS on MPTP opening was confirmed in vitro too.

As nonspecific pores, the exact components of MPTP are not fully understood. Although MPTP is modulated by cyclophilin D, Voltage-Dependent Anion Channel (VDAC), and Phosphate Carrier [25-27], recent studies found that the main modulate component of MPTP is the c-ring of ATP synthase, the number of which was determined by the expression of c-subunit $[11,12,28]$. The ring of $c$ subunits constitutes the membrane domain which were shared by both MPTP and ATP synthase. Azarashvili et al. [29] demonstrated that MPTP formation is associated with de novo assembly of channels comprising c-subunit, polyhydroxybutyrate, and inorganic polyphosphate, which is independent of ATP synthase. By contrast, He et al. found 
persistence of the mitochondrial permeability transition in the absence of subunit $c$ of human ATP synthase [13]. Csubunit knockout human HAP1 and 143B cells preserves the characteristic properties of the permeability transition pore. Given in the opposite results obtained from 293T cells and rat cortical neurons $[12,25]$, whether $\mathrm{c}$-subunit is the component of MPTP in human cells and whether c-subunit knockout cells have alternative means to develop permeability still needed to be further researched.

In the present study, our results showed that c-subunit of ATP synthase was downregulated with DSS treatment under IR scenario both in vivo and in vitro. This indicates that inhibition of c-subunit expression can alleviate IR injury. Regardless of the relationship between c-subunit and MPTP, c-subunit is the potential target to treat IR.

ATP subunit has three isoforms, encoded by ATP5G1, ATP5G2, and ATP5G3 genes, respectively [25]. Mammalian c-subunit consists of a mature protein with 76 amino acids, which is identical between all three isoforms. All these three c-subunit isoforms are functional and contribute to the $\mathrm{F}_{0}$ structure, but they have variable expression patterns in different tissues. For example, ATP 5G1 was abundantly expressed in heart [30].

Cardiomyocytes are sensitive to the changes of microenvironment, especially ischemia. Numerous studies have reported that mRNA transcription and protein expression change during the first 90 minutes of ischemic-reperfusion episode in both human and animals [31,32]. ATP 5G1, which target to mitochondria, can be imported into mitochondria under ischemic conditions [10, 29].

In vitro experiment shows that the cardioprotective effect of DSS disappeared under circumstance of ATP 5G1 overexpression cells. These results suggested that the effect of DSS might be through regulating ATP 5G1 gene expression. However, our study had limitations that the specific pathway related to ATP 5G1 needs to be further investigated. Also, the respiratory activity of the DSS-treated mitochondria should also be evaluated; it has been suggested that mitochondria may be less leaky to protons in the absence of the c-subunit [13]. In addition, excessive c-subunit will form hydrophobic aggregates (such as in Batten disease) that increase mitochondrial coupling of oxygen [33]. Therefore, it is interesting to know whether reduction of c-subunit expression improves the mitochondrial ATP bioenergetics.

There are also studies that support the role of c-subunit in MI treatment. Campo et al. enrolled 158 patients with first episode of acute anterior ST-segment elevation myocardial infarction (STEMI) who were treated successfully with percutaneous coronary intervention (PCI) [34]. They provided the first evidence that c-subunit is detectable in the serum of STEMI patients, but the data did not follow normal distribution (median 6.3\% [4-9.3\%]; range 1.1-47.5\%). In humans, the roles of MPTP and c-subunit are strongly influenced by several confounding factors, including thrombolysis application in myocardial infarction, myocardial perfusion grade, thrombolysis in myocardial infraction (TIMI) frame court, extent of ST-segment resolution, and presence of cardiac marker. These findings help us to explain why drugs to treat reperfusion injury always failed in randomized clinical trials.

\section{Prospective}

Herein, DSS was shown both in vivo and in vitro that can protect cardiomyocytes from apoptosis and inhibit MPTP opening in IR injury. Expression of c-subunit of ATP synthase was demonstrated to decline in both mRNA and protein level with DSS administration in IR background. The protection effect of DSS recedes with ATP 5G1 overexpression, which indicates its role in DSS signaling.

The limitation of the present study needs to be improved in the future research. Firstly, the drug effect on subunit $c$ might be mediated by an effect on the whole ATP complex. Whether DSS can alter other subunits of ATP synthase is not clear at present. In addition, the underlying mechanism of the curative effect of DSS is related to inhibition of c-subunit expression which needs to be confirmed in human.

\section{Conflicts of Interest}

The authors do not have any conflicts of interest with the content of the paper. In addition, they do not have direct financial relation with any commercial identity mentioned in this article.

\section{Authors' Contributions}

Qing Gao and JingYi Zhao contributed equally to this work.

\section{Acknowledgments}

This work was supported by the National Natural Science Foundation of China (81503504, 81573733, and 81704004) and National Undergraduate Training Program for Innovation and Entrepreneurship (201510063005).

\section{References}

[1] H. K. Eltzschig and T. Eckle, "Ischemia and reperfusion-from mechanism to translation," Nature Medicine, vol. 17, no. 11, pp. 1391-1401, 2011.

[2] J. Q. Kwong and J. D. Molkentin, "Physiological and Pathological Roles of the Mitochondrial Permeability Transition Pore in the Heart," Cell Metabolism, vol. 21, no. 2, pp. 206-214, 2015.

[3] A. P. Halestrap and A. P. Richardson, "The mitochondrial permeability transition: a current perspective on its identity and role in ischaemia/reperfusion injury," Journal of Molecular and Cellular Cardiology, vol. 78, pp. 129-141, 2015.

[4] P. Bernardi, A. Rasola, M. Forte, and G. Lippe, "The mitochondrial permeability transition pore: channel formation by F-ATP synthase, integration in signal transduction, and role in pathophysiology," Physiological Reviews, vol. 95, no. 4, pp. 11111155, 2015.

[5] P. Bernardi and F. Di Lisa, "The mitochondrial permeability transition pore: molecular nature and role as a target in cardioprotection," Journal of Molecular and Cellular Cardiology, vol. 78, pp. 100-106, 2015.

[6] C. P. Baines, R. A. Kaiser, T. Sheiko, W. J. Craigen, and J. D. Molkentin, "Voltage-dependent anion channels are dispensable for mitochondrial-dependent cell death," Nature Cell Biology, vol. 9, no. 5, pp. 550-555, 2007. 
[7] J. E. Kokoszka, K. G. Waymire, S. E. Levy et al., "The ADP/ATP translocator is not essential for the mitochondrial permeability transition pore," Nature, vol. 427, no. 6973, pp. 461-465, 2004.

[8] M. Juhaszova, S. Wang, D. B. Zorov et al., "The identity and regulation of the mitochondrial permeability transition pore: Where the known meets the unknown," Annals of the New York Academy of Sciences, vol. 1123, pp. 197-212, 2008.

[9] A. Krauskopf, O. Eriksson, W. J. Craigen, M. A. Forte, and P. Bernardi, "Properties of the permeability transition in $\mathrm{VDACl}^{-/-}$mitochondria," Biochimica et Biophysica Acta (BBA) - Bioenergetics, vol. 1757, no. 5-6, pp. 590-595, 2006.

[10] M. Bonora, A. Bononi, E. De Marchi et al., "Role of the c subunit of the FO ATP synthase in mitochondrial permeability transition," Cell Cycle, vol. 12, no. 4, pp. 674-683, 2013.

[11] K. N. Alavian, G. Beutner, E. Lazrove et al., "An uncoupling channel within the c-subunit ring of the F1FO ATP synthase is the mitochondrial permeability transition pore," Proceedings of the National Acadamy of Sciences of the United States of America, vol. 111, no. 29, pp. 10580-10585, 2014.

[12] S. Nesci, F. Trombetti, V. Ventrella, and A. Pagliarani, "The c-Ring of the F1FO-ATP Synthase: Facts and Perspectives," Journal of Membrane Biology, vol. 249, no. 1-2, pp. 11-21, 2016.

[13] J. He, H. C. Ford, J. Carroll, S. Ding, I. M. Fearnley, and J. E. Walker, "Persistence of the mitochondrial permeability transition in the absence of subunit c of human ATP synthase," Proceedings of the National Acadamy of Sciences of the United States of America, vol. 114, no. 13, pp. 3409-3414, 2017.

[14] C. Fernandez-Sanz, M. Ruiz-Meana, J. Castellano et al., "Altered FoF1 ATP synthase and susceptibility to mitochondrial permeability transition pore during ischaemia and reperfusion in aging cardiomyocytes," Thrombosis and Haemostasis, vol. 113, no. 3, pp. 441-451, 2015.

[15] Z. Qiao, J. Ma, and H. Liu, "Evaluation of the antioxidant potential of salvia miltiorrhiza ethanol extract in a rat model of ischemia-reperfusion injury," Molecules, vol. 16, no. 12, pp. 10002-10012, 2011.

[16] Y. Yin, Y. Guan, J. Duan et al., "Cardioprotective effect of Danshensu against myocardial ischemia/reperfusion injury and inhibits apoptosis of H9c2 cardiomyocytes via Akt and ERK1/2 phosphorylation," European Journal of Pharmacology, vol. 699, no. 1-3, pp. 219-226, 2013.

[17] Y. Meng, W.-Z. Li, Y.-W. Shi, B.-F. Zhou, R. Ma, and W.-P. $\mathrm{Li}$, "Danshensu protects against ischemia/reperfusion injury and inhibits the apoptosis of $\mathrm{H} 9 \mathrm{c} 2$ cells by reducing the calcium overload through the p-JNK-NF- $\kappa$ B-TRPC6 pathway," International Journal of Molecular Medicine, vol. 37, no. 1, pp. 258-266, 2016.

[18] G. Fan, J. Yu, P. F. Asare et al., "Danshensu alleviates cardiac ischaemia/reperfusion injury by inhibiting autophagy and apoptosis via activation of mTOR signalling," Journal of Cellular and Molecular Medicine, vol. 20, no. 10, pp. 1908-1919, 2016.

[19] R. M. Bell, M. M. Mocanu, and D. M. Yellon, "Retrograde heart perfusion: the Langendorff technique of isolated heart perfusion," Journal of Molecular and Cellular Cardiology, vol. 50, no. 6, pp. 940-950, 2011.

[20] P. Liao, G. Sun, C. Zhang et al., "Bauhinia championii flavone attenuates hypoxia-reoxygenation induced apoptosis in $\mathrm{H} 9 \mathrm{c} 2$ cardiomyocytes by improving mitochondrial dysfunction," Molecules, vol. 21, no. 11, article no. 1469, 2016.

[21] W.-X. Sun, H.-Y. Zheng, and J. Lan, "Edaravone protects osteoblastic cells from dexamethasone through inhibiting oxidative stress and mPTP opening," Molecular and Cellular Biochemistry, vol. 409, no. 1-2, pp. 51-58, 2015.

[22] G. Morciano, C. Giorgi, M. Bonora et al., "Molecular identity of the mitochondrial permeability transition pore and its role in ischemia-reperfusion injury," Journal of Molecular and Cellular Cardiology, vol. 78, pp. 142-153, 2015.

[23] L. Feng, S. Fu, W. Du et al., "Validation and application of an rapid HPLC-MS method for the determination of salvianic acid a in human plasma," Journal of Chromatographic Science (JCS), vol. 53, no. 5, pp. 771-777, 2015.

[24] J. Yu, L. Wang, M. Akinyi, Y. Li, Z. Duan, Y. Zhu et al., "Danshensu protects isolated heart against ischemia reperfusion injury through activation of Akt/ERK1/2/Nrf2 signaling," International Journal of Clinical and Experimental Medicine, vol. 8, pp. 14793-14804, 2015.

[25] L. K. Seidlmayer, V. V. Juettner, S. Kettlewell, E. V. Pavlov, L. A. Blatter, and E. N. Dedkova, "Distinct mPTP activation mechanisms in ischaemia-reperfusion: Contributions of $\mathrm{Ca}^{2+}$, ROS, $\mathrm{pH}$, and inorganic polyphosphate," Cardiovascular Research, vol. 106, no. 2, pp. 237-248, 2015.

[26] M. Bonora, C. Morganti, G. Morciano, C. Giorgi, M. R. Wieckowski, and P. Pinton, "Comprehensive analysis of mitochondrial permeability transition pore activity in living cells using fluorescence-imaging-based techniques," Nature Protocols, vol. 11, no. 6, pp. 1067-1080, 2016.

[27] K. Shintani-Ishida and K.-I. Yoshida, "Mitochondrial mcalpain opens the mitochondrial permeability transition pore in ischemia-reperfusion," International Journal of Cardiology, vol. 197, Article ID 20666, pp. 26-32, 2015.

[28] M. R. Alam, D. Baetz, and M. Ovize, "Cyclophilin D and myocardial ischemia-reperfusion injury: A fresh perspective," Journal of Molecular and Cellular Cardiology, vol. 78, pp. 80-89, 2015.

[29] T. Azarashvili, I. Odinokova, A. Bakunts et al., "Potential role of subunit $\mathrm{c}$ of F0F1-ATPase and subunit $\mathrm{c}$ of storage body in the mitochondrial permeability transition. Effect of the phosphorylation status of subunit c on pore opening," Cell Calcium, vol. 55, no. 2, pp. 69-77, 2014.

[30] A. Pandini, J. Kleinjung, W. R. Taylor, W. Junge, and S. Khan, "The Phylogenetic Signature Underlying ATP Synthase c-Ring Compliance," Biophysical Journal, vol. 109, no. 5, pp. 975-987, 2015.

[31] Y. Zhu, J. Wu, and S.-Y. Yuan, "MCT1 and MCT4 expression during myocardial ischemic-reperfusion injury in the isolated rat heart," Cellular Physiology and Biochemistry, vol. 32, no. 3, pp. 663-674, 2013.

[32] L. A. Saddic, S. M. Nicoloro, O. T. Gupta et al., "Joint analysis of left ventricular expression and circulating plasma levels of Omentin after myocardial ischemia," Cardiovascular Diabetology, vol. 16, no. 1, 2017.

[33] V. Havlíčková, V. Kaplanová, H. Nůsková, Z. Drahota, and J. Houštěk, "Knockdown of F1 epsilon subunit decreases mitochondrial content of ATP synthase and leads to accumulation of subunit c," Biochimica et Biophysica Acta (BBA) - Bioenergetics, vol. 1797, no. 6-7, pp. 1124-1129, 2010.

[34] G. Campo, G. Morciano, R. Pavasini et al., "Fo ATP synthase C subunit serum levels in patients with ST-segment Elevation Myocardial Infarction: Preliminary findings," International Journal of Cardiology, vol. 221, pp. 993-997, 2016. 


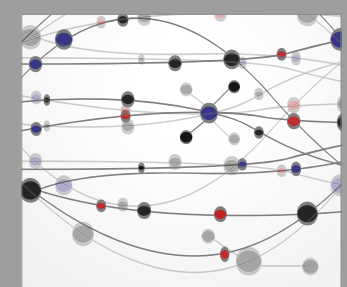

The Scientific World Journal
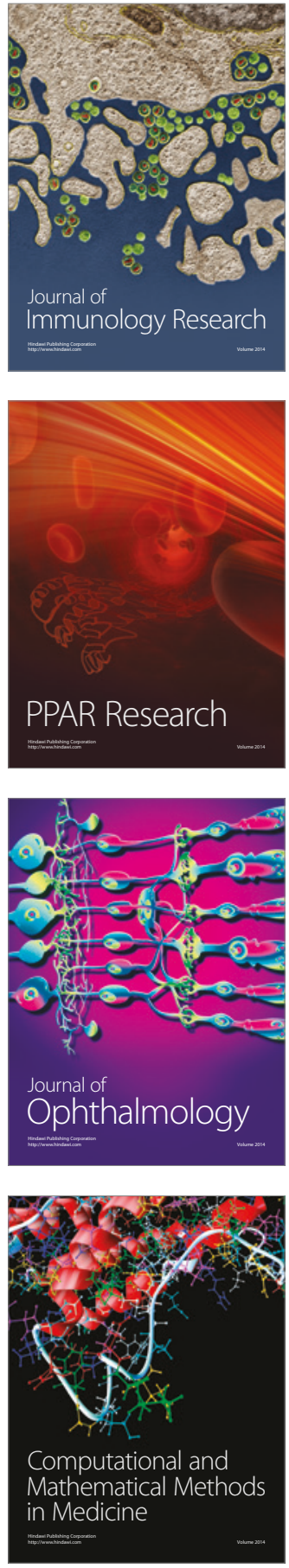

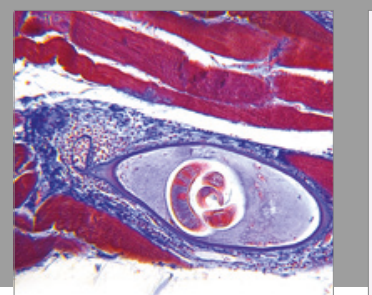

Gastroenterology Research and Practice
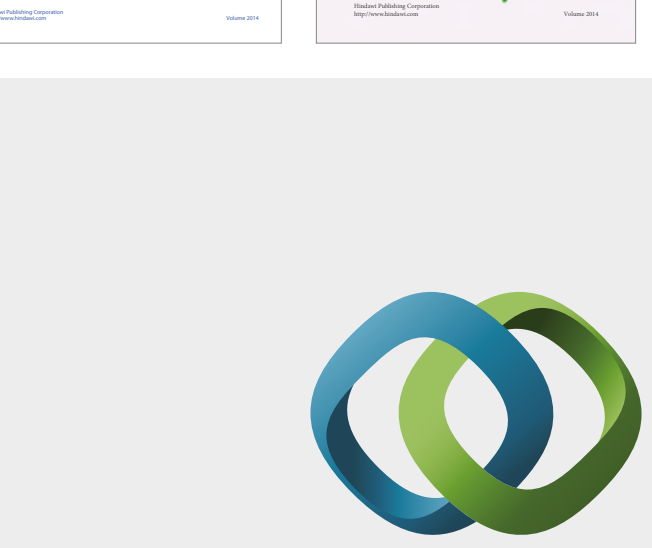

\section{Hindawi}

Submit your manuscripts at

https://www.hindawi.com
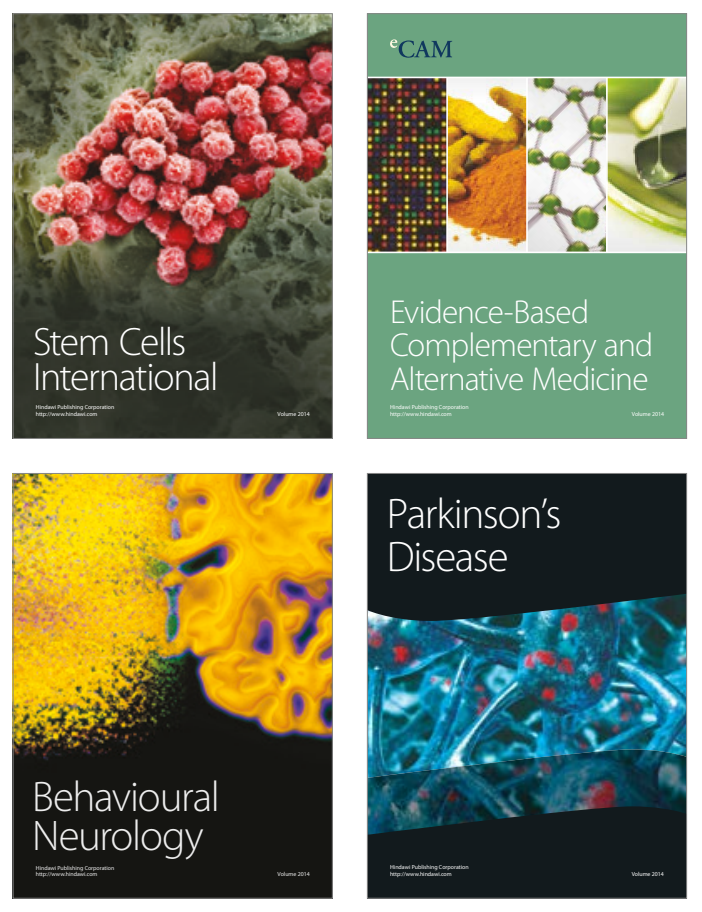
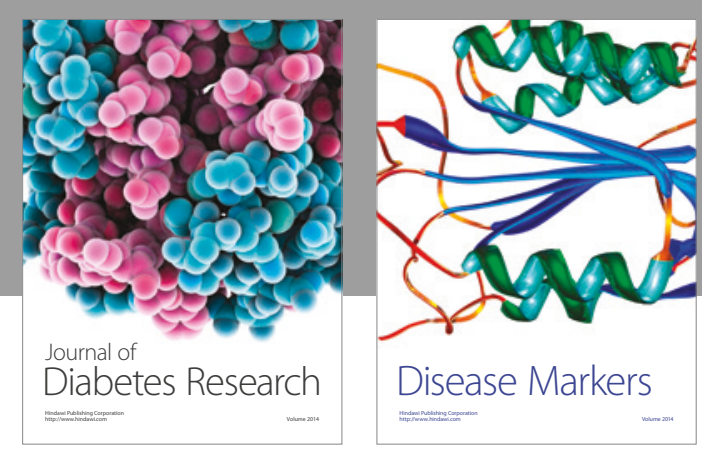

Disease Markers
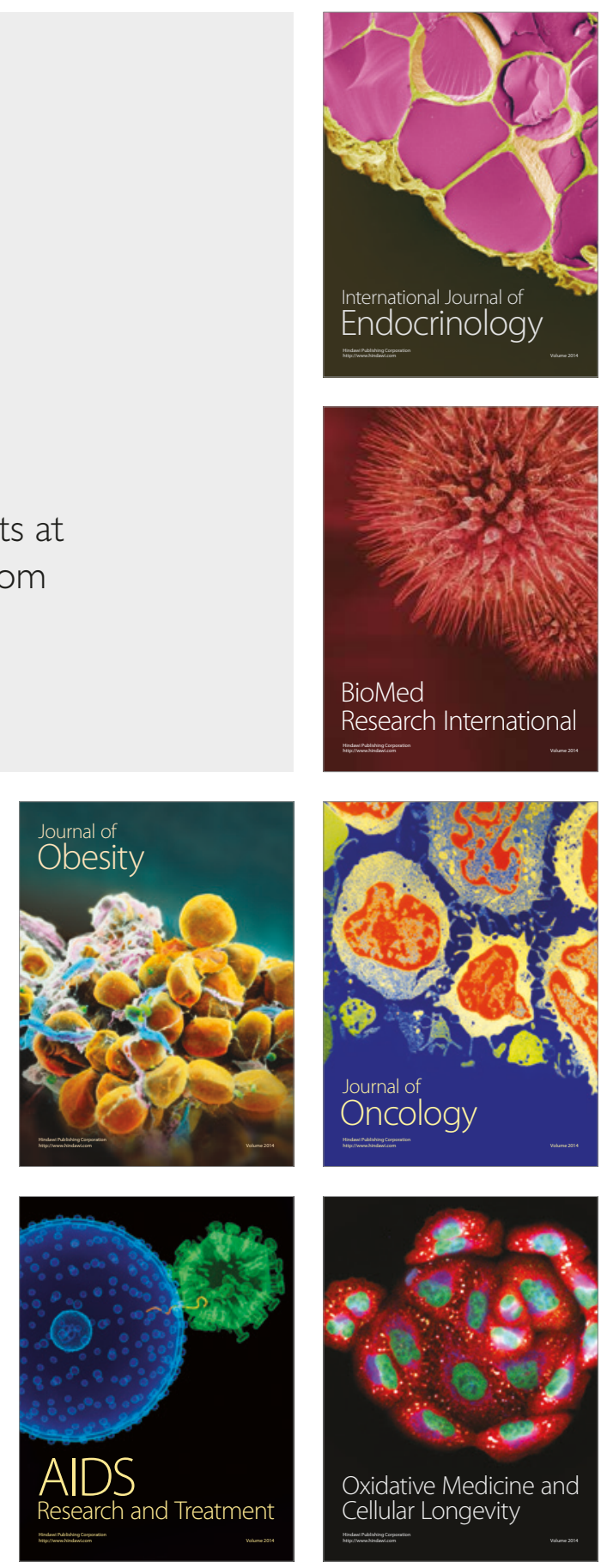